\title{
BMJ Open Active Play in After-school Programmes: development of an intervention and description of a matched-pair cluster- randomised trial assessing physical activity play in after-school programmes
}

Kirsti Riiser, ${ }^{1}$ Sølvi Helseth, ${ }^{2}$ Hanna Ellingsen, ${ }^{3}$ Bjørg Fallang, ${ }^{1}$ Knut Løndal ${ }^{4}$

To cite: Riiser K, Helseth S, Ellingsen $\mathrm{H}$, et al. Active Play in After-school Programmes: development of an intervention and description of a matchedpair cluster-randomised trial assessing physical activity play in after-school programmes. BMJ Open 2017;7:e016585. doi:10.1136/ bmjopen-2017-016585

- Prepublication history for this paper is available online. To view these files please visit the journal online (http://dx.doi. org/10.1136/bmjopen-2017016585).

Received 24 February 2017 Revised 26 May 2017 Accepted 14 June 2017

\section{CrossMark}

${ }^{1}$ Department of Physiotherapy, Faculty of Health, Oslo and Akershus University College of Applied Sciences, Oslo, Norway ${ }^{2}$ Department of Nursing and Health Promotion, Faculty of Health, Oslo and Akershus University College of Applied Sciences, Oslo, Norway ${ }^{3}$ Department of Family Health Services, Municipality of Sandefjord, Sandefjord, Norway ${ }^{4}$ Department of Primary and Secondary Teacher Education, Faculty of Education and International Studies, Oslo and Akershus University College of Applied Sciences, Oslo, Norway

Correspondence to

Dr Kirsti Riiser;

kirsti.riiser@hioa.no

\section{ABSTRACT}

Introduction Interventions delivered in after-school programmes (ASPs) have the potential to become a means of ensuring adequate physical activity among schoolchildren. This requires a motivational climate, allowing for self-determined play. If trained, ASP staff may represent a valuable resource for supporting such play. Increasing knowledge and supportive skills among ASP staff may also potentially increase their motivation for work. The purpose of this article is to describe the development of the 'Active Play in ASP' intervention, which aims to promote physical activity among first graders attending ASP, and to present a protocol for a matched-pair cluster-randomised trial to evaluate the intervention.

Methods and analysis Informed by experiences from practice, evidence-based knowledge and theory, the intervention was developed in a stepwise process including focus group meetings and a small-scale pilot test. The intervention contains a course programme for ASP staff to increase their skills in how to support physical activity through play. In a cluster randomised controlled trial, the ASPs will be matched and randomly allocated to receive the 7-month intervention or to a control group. Outcomes will be assessed at baseline, after 7 and 19 months. First graders attending the ASPs included are eligible. The primary outcome will be accelerometerdetermined minutes in moderate to vigorous physical activity in the ASP. The study uses a mixed methods approach including observations and interviews to provide rich descriptions of the concept of children's physical activity in ASP. Moreover, the trial will assess whether the ASP staff benefits from participation in the intervention in terms of increased work motivation. Lastly, process evaluations of programme fidelity, satisfaction and suggestions on improvement will be performed.

Ethics and dissemination The study is approved by the Data Protection Official for Research (reference no 46008). Results will be presented in conferences and peer-reviewed journals.

Trial registration number Clinical Trials (NCT02954614), pre-results.
Strengths and limitations of this study

- The Active Play in ASP is the first randomised controlled physical activity study that is performed in an after-school programme (ASP) setting in Scandinavia.

- The study will apply a mixed methods approach using accelerometers, observations and interviews to assess physical activity, providing an extensive insight into children's physical activity in ASP.

- A weakness may be that the intervention follow-up throughout the school year is limited to one meeting per month. The decision is made pragmatically due to a consideration of what is realistic should the intervention be translated into routine practice.

- Using local school physiotherapists to deliver parts of the intervention strengthens the external validity of the study, but may also increase variation in the results.

\section{BACKGROUND}

The relationships between physical activity and children's health and well-being are widely acknowledged. Physical activity may positively influence a number of health factors. ${ }^{12}$ Research has also begun to emphasise the role played by children's physical-motor functioning and activity levels in academic performance, ${ }^{34}$ as well as its effect as a preventive mechanism against antisocial behaviour. ${ }^{5}$ Another important reason for focusing on children's physical activity levels is the preventive effect physical activity may have on overweight and obesity. ${ }^{6}$ Perhaps most importantly, physical activity may be a positive source for the development of children's well-being. ${ }^{5}$ However, as shown in research from the field of sports and physical education, in order to increase wellbeing, an autonomy supportive and mastery oriented motivational climate is required, allowing for the child's self-determination and 
the intrinsic values of the activity and the activity's character of play. ${ }^{5}$ In the present context, the term 'physical activity play' refers to such play, incorporating subjective and experienced aspects of movements and self-driven and autotelically oriented activities. ${ }^{78}$ Physical activity play includes vigorous locomotory movements, stabilising postures and/ or manipulative movements. ${ }^{8}{ }^{9}$ Physical activity, which is commonly described as any bodily movement produced by skeletal muscles that result in energy expenditure, ${ }^{10}$ can take place in the household or domestic domain, the occupational domain, the transportation domain and the leisure time domain. ${ }^{11}$ Physical activity is thus considered a collective term including physical activity play as well as for example, hiking or more organised forms of sports activities.

There is some evidence that physical activity interventions in school can be effective in increasing the proportion of children engaging in moderate and vigorous physical activity (MVPA) during school time as well as the duration of time spent on these activities. ${ }^{12}$ However, physical activity in school is often limited to physical education or recesses. Consequently, during school hours, the children are not provided with opportunities to be as physically active as recommended, that is, at least 1 hour of moderate to vigorous physical activity a day. ${ }^{13}$ Interventions directed at after-school programmes (ASPs) have the potential to become a means of increasing physical activity among young children. ${ }^{14}$ Previous research has indicated that ASP interventions emphasising competence building among the staff can lead to increased levels of physical activity for the children. ${ }^{15-18}$ The studies indicate that effective programmes should emphasise positive feedback and encouragement regarding physical activity, goal setting and evaluation of measures, development of schedules for physical activity, structuring and administration of the environment and arrangements for physical activity for the children. The present study builds on these findings by investigating a course programme for increasing supportive skills and knowledge about children's play among ASP staff. No national educational objectives are associated with Norwegian ASPs. In contrast with the sports-dominated extracurricular physical education in several other European countries, ${ }^{19}$ Norwegian ASPs are expected to stimulate self-managed activities in the children's leisure time. ${ }^{20}$ Thus, the stage is set to provide various content appropriate to the interests of the children, for example, various types of physical activity. As $62 \%$ of first to fourth graders and as many as $81 \%$ of first graders attend ASP, a large proportion of children in the relevant age group can be reached. Results from previous research in Norway show that children's physical activity during their stay in the ASP is extensive when they have time devoted to child-managed play outdoors. ${ }^{20} 21$ Nevertheless, some children fall by the wayside, and this may hamper their activity level and their well-being. ${ }^{22}$ It also seems to be a trend that activities in ASPs are more organised than earlier. ${ }^{23}$ The staff are more engaged in arranging and managing various activities for groups of children, and their opportunities to attend to child-managed activities have diminished. This has weakened their possibility to initiate child-managed movement play among the least active children. ${ }^{23}$ It seems to be particularly important for the ASP staff to develop pedagogic skills in order to provide adapted frameworks for all children's physical activity, in addition to providing child-managed physical activity play. ${ }^{25}$ Thus, it is essential to know how to support such play. In Norway, only a minority of the employees in ASPs has formal pedagogical education, and there seems to be a lack of competence in how to approach and engage in children's play. ${ }^{26}$ If trained, ASP staff members may represent a valuable resource for supporting physical activity play and other forms of physical activities in everyday life for all children. Another potential benefit of an intervention addressing increased knowledge and skills among ASP staff is that the staff may experience a boost in their work motivation. This has previously been shown to be the case among physical education teachers. ${ }^{27}$ Physiotherapists have an essential role in the delivery of primary healthcare to children and adolescents in Norway. ${ }^{28}$ Within a school health context the physiotherapist initiates and participates in tasks focusing on health promotion, disease prevention and interventions that improve or maintain fitness, health and well-being. Their role includes provision of education and consultation with other professionals in the child's environment, making physiotherapists important contributors to an ASP based physical activity intervention. Few, if any, studies have evaluated efforts concerning the use of physical activity play as a health-promoting strategy involving school physiotherapists.

\section{AIM}

The purpose of this article is to describe the development of the Active Play in ASP intervention and to present a protocol for a matched-pair cluster-randomised trial. The Active Play in ASP intervention comprises a course programme for increasing knowledge and supportive skills among ASP staff. The aim of the planned trial is to assess the immediate and long-term (1 year after the intervention ends) efficacy of the intervention on first graders' physical activity in the ASP and their well-being, conceptualised here as quality of life. Moreover, we aim to investigate the characteristics of first graders' physical activity in ASP and the qualitative aspects of their understanding and experience of the activity. In addition, the trial will explore if the ASP staff can benefit from participation in the intervention in terms of increased motivation and work satisfaction. Lastly, we will perform a process evaluation of the intervention.

\section{METHODS AND ANALYSIS}

\section{Development of the intervention}

In the first phase of the Active Play in ASP intervention development, we gathered information from the field, identified the evidence base and chose appropriate theory (figure 1). 


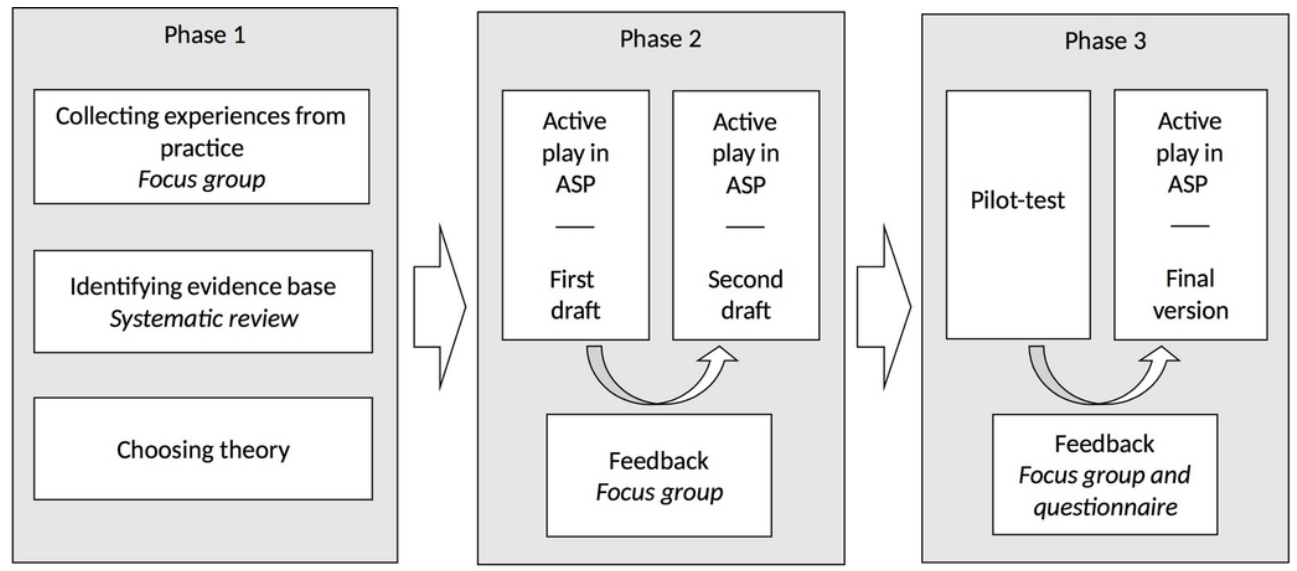

Figure 1 Process of development of Active Play in ASP. ASP, after-school programme.

As emphasised by Craig $e t a l,{ }^{29}$ a key question in the development and evaluation of complex interventions is whether the intervention will work in everyday practice. In the present study, we draw on experiences from 'Health Promoting ASP', a project previously run in five ASPs in a municipality in Norway. The project emphasises healthy food, physical activity and well-being among the children during ASP time. It was initiated by local school physiotherapists in cooperation with school headmasters and implemented throughout a school year. The project has been well received by the ASP staff and the school administrations. However, the project is insufficiently evaluated, which makes it difficult to determine the impact on the children's behaviour. In the present trial, we decided to limit the scope of the intervention and focus solely on how to support physical activity. A school physiotherapist from 'Health Promoting ASP' and three employees representing three different ASPs participated in a semistructured focus group meeting to share their experiences and to pinpoint possible barriers to and facilitators for implementation and potential successful outcomes. The focus group meeting was moderated by one of the researchers. Main features of the Active Play in ASP intervention, both content and structure, were outlined based on the summary of the focus group meeting.

Parallel to this process, previous research on physical activity interventions in ASPs was systematically reviewed and published in a master thesis. ${ }^{30}$ The review, which included 17 articles, found positive effects on the children's activity level only in interventions that incorporated flexible programmes that were adaptable to each single ASP. Highly structured programmes (ie, standardised activity programmes) were reported to be more difficult to implement, which may explain their limited effect on children's physical activity. ${ }^{31-33}$ The results of the review echoed the feedback given by the focus group, which also emphasised the value of an adaptable intervention. The focus group members stressed that it is essential to develop an understanding of how each ASP is organised. Contextual factors and professional experiences need to be acknowledged and included in the implementation process.
In this first phase, we also decided on a theoretical framework. Self-determination theory (SDT) is frequently used in health behaviour research as well as in educational research and was considered appropriate in the context of children's activity play. The theory has relevance for understanding motivated physical activity engagement. It emphasises that being motivated by self-determined reasons leads to greater engagement and well-being than being motivated by controlled reasons. ${ }^{34}$ Self-determined motivation is associated with positive outcomes in children, such as exercise behaviour, quality of life and a positive self-concept. ${ }^{35}$ According to SDT, social environments that support the individual's basic psychological needs (autonomy, competence and relatedness) will foster more self-determined motivation. ${ }^{36}$ Autonomy reflects the need to engage in activities with a sense of choice, competence represents the feeling that one will be able to accomplish tasks, while relatedness refers to the sense of being understood and respected by significant others. ${ }^{37}$ Autonomy support, structure and interpersonal involvement can support the basic psychological needs and thus facilitate adoption and maintenance of physical activity. ${ }^{38}$ Facilitating the children's choices and supporting their free expression are central to basic need support in play. In an ASP context, application of these principles implies that the staff should not intervene in play situations in a commanding or controlling manner, but rather support and gently encourage activities. Simultaneously, the selfchosen and child-managed character of play should be retained. ${ }^{39}$ In addition to informing the content of the present intervention, for example, application of theoretically anchored principles for activity support, the SDT has contributed to the modelling of the likely processes of change. ${ }^{40}$

In the second phase of development, we drafted a course programme that subsequently was presented to the same ASP focus group that participated in the initial phase. The group was encouraged to respond to questions regarding the feasibility and usefulness of the intervention. A second draft was prepared building on their feedback. In the third phase, the intervention was tested in a small-scale pilot study including two ASPs over a period 
of 4 months. Along with the piloting of the intervention, we tested all outcome measures and measurement procedures at baseline and postintervention. The staff from the two pilot ASPs provided feedback by answering a short questionnaire with semi-structured questions related to their experience of the intervention. In addition, a strategic sample of three employees from each of the two ASPs participated in two focus group interviews moderated by one of the researchers. The focus group interview allowed the employees to speak more freely about their experiences with the intervention. Only minor changes had to be made to complete the final version.

\section{Intervention content}

Active Play in ASP is a 7-month course programme (October-May) aimed at ASP staff with the intention of increasing their knowledge and skills regarding how to support children's physical activity play. However, providing activity support is not merely the responsibility of the employee in interaction with one child or group of children. The programme also emphasises the potentials of institutional activity support, reflected in how the ASP is organised concerning time structure (time spent indoors/outdoors), routines and rules, and the ASP's access to and utilisation of activity places and equipment. The intervention has the potential to reach all children in the ASP. However, as described later, only first graders are included in the measurements of the trial.

The ASP staff in each intervention ASP will participate in the course programme as described below (table 1). The initial part of the programme is led by the researchers. The local school physiotherapist attends and contributes during the initial part (the introductory sessions, mapping and planning) and is responsible for the fivemonthly follow-up meetings after the first sessions. Thus, prior to the ASP course programme, the physiotherapists are provided with an 8-hour introduction course presenting the intervention and how it is organised, emphasising their role. To increase fidelity and adherence to the intervention, the physiotherapists receive a detailed workbook outlining the interventions' rationale, content and assignments for the ASP staff.

The ASP course programme starts with two 3-hour sessions arranged locally at each participating ASP within a period of 2 weeks. All staff will attend. The sessions focus on children's physical activity in play, friends, activity place, ASP staff's interaction styles, motivation and activity support. The sessions include lectures, theme-based discussions and group tasks. The staff are encouraged to give examples from their own practical experience. Moreover, in a separate meeting the ASP is mapped to document activity equipment and indoor and outdoor facilities. This information is used as a supplement in the following meetings. Subsequently, the staff, supervised by the local school physiotherapist and a research group member, outline how the ASP will include new knowledge and previous experiences in strategies for supporting children's activity play during their time in the ASP. The programme continues during the school year with monthly meetings for the staff and the local school physiotherapist where they work on predefined tasks related to physical activity play (table 1 ). Participation in the intervention and the study will not involve any additional costs for the ASPs.

In line with the basic principles of SDT, we also aim to create a supportive context for the staff during the course programme. By providing a meaningful rationale

Table 1 Intervention components and course programme content

\begin{tabular}{lll}
\hline & Component & Content \\
\hline $\begin{array}{l}\text { Introductory for } \\
\text { schoolphysiotherapists }\end{array}$ & 1-day course & $\begin{array}{l}\text { Information on the intervention and the physiotherapists' role } \\
\text { and responsibilities. Presentation of intervention workbook. }\end{array}$ \\
Introductory course for & &
\end{tabular}

Introductory course for school physiotherapists

Course programme ASP 3-hour session staff

3-hour session

Mapping

Planning (1-2 hour meeting)

Five meetings (monthly 1-2 hours) led by the local school physiotherapist
Introduce research-based knowledge about children's physical activity in play. Increase the staff's awareness of how such play can be influenced and supported in ASP.

Basic theoretical principles of SDT applied to physical activity and physical activity play among children; how to be activity supportive.

Thorough mapping of the ASP equipment and facilities.

Summary of introductory sessions; how to make use of new knowledge.

Discussions and practical tasks focusing:

- Motor learning in children

- Equipment and environment

- Mapping of staff competencies

- Inclusion/exclusion in play

- How to lead and support activity in groups

ASP, after-school programme; SDT, self-determination theory. 


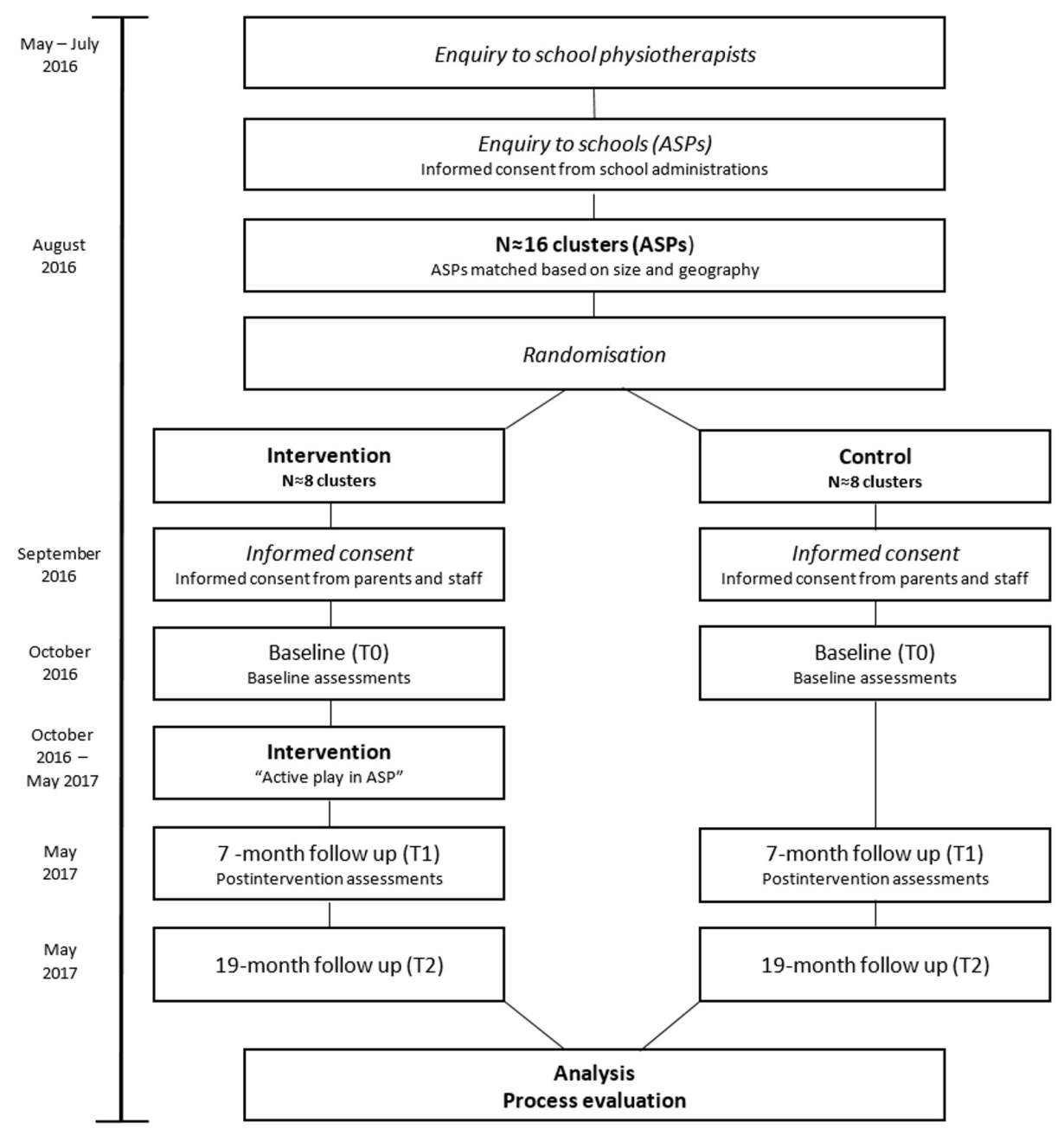

Figure 2 Flowchart of the study design. ASPs, after-school programmes.

for the intervention, acknowledge the staff's feelings and give opportunities for choice and contribution, their autonomy is supported. Structure is provided through informative feedback, clear expectations and optimal challenges while interpersonal involvement will be ensured by devoting time, energy and affection to the staff before, during and after the course sessions. ${ }^{41}{ }^{42}$ An overview of the trial procedure is outlined in figure 2 .

\section{Study design}

The study is designed as a matched-pair cluster-randomised trial using a mixed methods approach. The intervention group will receive the Active Play in ASP intervention while the control ASPs receive no follow-up in addition to the usual ASP. A process evaluation is embedded in the trial (figure 2).

\section{Recruitment}

The intervention follow-up and the trial rely on assistance from local school physiotherapists. Even though municipalities in Norway are strongly advised to ensure physiotherapy resources for health promotion activities in schools through the school health services, such resources are generally scarce. Thus, as a first step in the recruitment process, all school health services in centrally located municipalities (maximum 90min' drive from the study office) in three counties in the eastern part of Norway will be approached and invited to participate $(n \approx 45)$. As a sufficient number of school physiotherapists are located and have signed up, they are asked to assist in the further recruitment of ASPs in schools within their area of responsibility. This will provide us with a sample of schools willing to participate. School administrators are required to provide written consent to participation. The consent is obtained before randomisation and is considered binding. After randomisation, the parents of all first grade pupils (age 5-6 years) attending the participating ASPs are informed about the study and asked for a written consent on behalf of their child. The age group is chosen based on the fact that nearly every first grader in Norway attends ASP and that we have less information about physical activity in this group compared with older children. All ASP staff and physiotherapists will be asked for a written consent to participation in the trial. The control ASPs will be offered the intervention after the study is completed.

\section{Randomisation}

Prior to randomisation, the clusters, that is the ASPs in the schools, will be paired based on available background 
information on size and geography. The categories 'small', 'medium' or 'large' and 'urban' or 'rural' are chosen based on the assumption that the size of the school with regard to number of pupils as well as space and access to nature areas may have an impact on the children's activity level. Following matching, tags with the names of the ASPs are put in envelopes and sealed, and then randomly allocated to receive the intervention or to control. While the recruitment, enrolment of participants and the matching of clusters are done by the research team, the person revealing the allocation is not involved in the study. Due to the design of the study, a blinding of trial participants (ASP staff) and outcome assessors is not feasible.

\section{Measures}

Excepting the qualitative interviews and process evaluation performed in the intervention group post intervention, measures are obtained from both groups at three time points: at baseline (T0), immediately after the 7-month intervention (T1) and 1-year postintervention (T2). A timeline for the intervention study is shown in figure 2.

Because no measure is suitable for assessing both type, amount, intensity, variability, quality and experience of physical activity, several instruments and methods, quantitative as well as qualitative, will be used to capture as much information as possible. The primary outcome will be child physical activity intensity, which will be assessed objectively by ActiGraph accelerometer during the time spent in ASP over a period of 1 week. Following a standardised procedure, the accelerometers will be fitted to the child by one of the staff members at the time of arrival and removed before leaving for home. In order to detect the intermittent activity patterns of small children, the accelerometer will collect data at $10 \mathrm{~s}$ epochs. Minutes spent in MVPA, low physical activity and inactivity will be estimated with cut points with MVPA defined at equal to or above 2000 counts per minute, low activity between 100 and 1999 counts per minute and inactivity at less than 100 counts per minute. ${ }^{43}$ The length of time spent in the ASP will be accounted for. To supplement the accelerometer measurements, the schedule of the day, common activities (duration of different types of activities) and factors that may affect physical activity indoors and outdoors (number of staff, weather and special events) will be logged daily by ASP staff during the week of accelerometer measurements.

Moreover, a subsample will be directly observed during ASP time. Registrations of both quantified physical activity (type, intensity, duration and frequency) and rich descriptions of physical activity during a day in ASP will be performed. Finally, qualitative interviews will be performed postintervention with a subsample of two children from each cluster in the intervention group. This sample will be strategically chosen by the ASP leader. The interview will focus on the children's experiences with physical activity in the ASP.
Secondary outcomes include the child's experience of being in the ASP. Items are adjusted from a questionnaire from the Norwegian part of the Health Behaviour in School-aged Children study. ${ }^{44}$ The items are chosen based on how they correspond with key concepts of SDT. The questions are answered electronically by the child in cooperation with the parents. Furthermore, child well-being, in this study conceptualised as health-related quality of life, is assessed by the Kidscreen-27 proxy version and obtained electronically. ${ }^{45}$ Self-reported leisure time physical activity outside school and ASP will be measured by the UngKan2 questionnaire. This measure is widely used in national studies of child and youth physical activity, providing reference data for the present study. ${ }^{43}$ The questionnaires will be completed electronically at home during the week of accelerometer measurements. An email with an invitation to a survey is sent to the parents of each participating child. Except for the Kidscreen-27, which is a proxy instrument, the questions are answered by the children in cooperation with their parents. Additionally, in order to control for body mass, the children's height and weight will be measured and body mass index calculated. ${ }^{46}$ The local school nurse or school physiotherapist will be responsible for the measurements following a written procedure. Data on gender and age are collected.

For evaluation of if and how the intervention may benefit the ASP staff, self-report instruments will be used for assessing their work-related basic needs satisfaction, ${ }^{47}$ motivation for work, ${ }^{48}$ job satisfaction ${ }^{49}$ and subjective well-being. ${ }^{50}$ At baseline, the staff will also be asked to report age, sex and duration of employment in the current ASP.

A process evaluation will be performed at the end of the intervention. ${ }^{51}$ All ASP staff from the intervention ASPs will be asked to complete a short questionnaire including questions on the experience of participation, potential obstacles, gains and improvements. Contextual influences on the implementation, programme fidelity, potential adjustments to the intervention and the number of employees attending the meetings, will be recorded. Data will be supplemented by summaries from the meetings and reviews of the intervention documents. A convenience sample of three to five staff members from each cluster will be asked to participate in semistructured focus group interviews exploring views on impact of the intervention on the children, the ASP in general and on the staff. They are also asked questions regarding potential improvements. All physiotherapists will be invited to participate in a similar focus group.

\section{Sampling}

A rough estimate of the required sample size is based on the primary outcome physical activity as measured by ActiGraph accelerometer. Due to the exploratory nature of our study, we keep the significance level alpha at $1 \%$ and power at $90 \%$ to correct for multiple testing. All tests will be two sided. Based on the results of our pilot test and previous studies, ${ }^{1416}$ we consider 6 min increase in MVPA 
during ASP time to be of clinical importance, which represents $10 \%$ of the 1 hour of MVPA recommended by the guidelines. Based on the above, we estimate $\mathrm{N}$ to be 121 in each group without accounting for cluster effects. We plan to enrol 200 children in each group to secure sufficient power for additional analyses on cluster level. With an estimation of a minimum of 25 first graders in each ASP, we will have to include a maximum of 16 ASPs. Based on experiences from the pilot, we have reasons to assume that the majority of the parents will give their consent.

For the qualitative observations, a sample of three children from each cluster will be randomly drawn. Initially, the children are stratified based on gender to ensure equal distribution of boys and girls.

The children eligible for selection for the qualitative interviews will be in the intervention group. A roughly estimated sample size would be 16-20 children with 2-3 children from each ASP. A strategic sampling aimed at maximum variation according to gender and ethnicity is an appropriate sampling procedure.

The expected number of participating ASP staff depends on the size of the ASPs that accept the request for participation. A rough estimate is $8-10$ employees per ASP, yielding a sample of approximately 150 .

\section{Analysis}

The observations will be analysed and presented with descriptive statistics in addition to text summaries. The differences between the intervention group and the control group will be assessed by repeated measure analyses using linear mixed models for repeated measures as implemented in SPSS v. 24.0. This approach is flexible and it is possible to model the dependence between observations from the same individual. Intervention status and time period will be modelled as main effects while a cluster effect will be accounted for in the model as a random effect.

Information from the activity logs recorded by the ASP staff will be quantified and categorised to be included in analysis of whether contextual factors (weather, indoor/ outdoor, organised/unorganised physical activity) influence mean physical activity intensity.

Qualitative interviews and field notes from the observations will be analysed by systematic text condensation, implying a hermeneutic approach to data collection and analysis. ${ }^{52} 53$ The NVivo 10 software for qualitative analysis will be used. Process data will be summarised and the text will be analysed using simple content analysis. ${ }^{54}$

\section{Ethics}

The study is reviewed and approved by the Data Protection Official for Research (NSD). Informed consent to participate in the study is requested from the parents on behalf of the children. In addition, age adjusted oral information will be given to the young children. Participants are guaranteed full confidentiality. Consent to participate in the trial will also be obtained from the ASP staff and the physiotherapists.
Information about participant identities will be stored separately from the study results. Data are anonymised in all publications and reports of the study. Participant data are protected in accordance with NSD's guidelines.

\section{Dissemination}

Results from the study will be published in scientific peer-reviewed journals and master thesis. Reports written in lay language will be provided to all participating ASPs and school administrations when the study is completed. Any changes or additions to the protocol will be reported to the Norwegian Centre for Research Data and registered in clinicaltrials.gov. Authorship is granted to project group members and others that fulfil the authorship criteria recommended by the International Committee of Medical Journal Editors.

\section{DISCUSSION}

The apparent need for systematically developed physical activity interventions adaptable to Norwegian ASPs makes a strong case for the trial described. The article describes how a complex intervention to ensure physical activity play during ASP time is carefully developed in close cooperation with school physiotherapists and representatives from ASPs. That the intervention originates from practice, and that the practice experiences are combined with previous research within a theoretical framework, are among the advantages of this study. Involvement of appropriate users in the different stages of an intervention study is likely to result in a higher chance of producing implementable data. ${ }^{29}$

The present article also describes how the intervention will be explored in a matched-pair cluster-randomised trial. Strength of the planned trial is its combination of measures of physical activity. Interventions, whether they include physical activity as a primary or secondary outcome, tend to focus on the quantity of physical activity (duration, intensity and frequency), and not the quality. This study aims to mix objectively measured physical activity, logs and direct observations to be better able to give rich descriptions of the concept of children's physical activity in ASP. By including qualitative methods in the investigation, we gain information about the type of physical activity the children actually perform, where they perform the activity, with whom they spend time, and whether the activity is initiated and managed by the children themselves or by adults. Mixing methods in the same study may thus increase the possibility of evaluating the effect in addition to gaining an understanding of the mechanisms involved in the outcome of the intervention. ${ }^{55}$

\section{Trial status}

The intervention is ongoing with baseline data collection completed in October 2016. Short-term intervention (T1) data collection is due to be completed in June 2017 and long-term data in June 2018. The study was registered in Clinical Trials (NCT02954614) in October 2016, prior to start-up of the intervention. 
Acknowledgements We thank the ASP staff who have been involved in the development and piloting of the intervention and the trial. Their enthusiastic participation was decisive in the development of Active Play in ASP.

Contributors All the authors contributed to the study's conception, planning and design. KR and HE were responsible for drafting the intervention and managing the pilot trial. KR had primary responsibility for writing the paper in close collaboration with KL. HE, BF and SH participated in revising the article by providing comments and revisions. All authors approved the final version for publication.

Funding This project is funded by the Norwegian Fund for Postgraduate Training in Physiotherapy and Oslo and Akershus University College of Applied Sciences.

Competing interests None declared.

Patient consent Obtained.

Ethics approval The study was first reviewed by the Regional Committee for Medical and Health Research Ethics. The Committee concluded that the study is not covered by the Health Research Act. Consequently, the study protocol was submitted and reviewed by The Data Protection Official for Research (NSD) to ensure that the project is in accordance with the Personal Data Act and the Personal Health Data Filing System Act (reference number 46008).

Provenance and peer review Not commissioned; externally peer reviewed.

Data sharing statement Once the study is completed, we will publish all relevant results. Unpublished results could be made available on request by contacting the authors.

Open Access This is an Open Access article distributed in accordance with the Creative Commons Attribution Non Commercial (CC BY-NC 4.0) license, which permits others to distribute, remix, adapt, build upon this work non-commercially, and license their derivative works on different terms, provided the original work is properly cited and the use is non-commercial. See: http://creativecommons.org/ licenses/by-nc/4.0/

(c) Article author(s) (or their employer(s) unless otherwise stated in the text of the article) 2017. All rights reserved. No commercial use is permitted unless otherwise expressly granted.

\section{REFERENCES}

1. Janssen I, Leblanc AG. Systematic review of the health benefits of physical activity and fitness in school-aged children and youth. Int $J$ Behav Nutr Phys Act 2010;7:40.

2. Biddle SJ, Gorely T, Stensel DJ. Health-enhancing physical activity and sedentary behaviour in children and adolescents. J Sports Sci 2004;22:679-701.

3. Biddle SJ, Asare M. Physical activity and mental health in children and adolescents: a review of reviews. Br J Sports Med 2011;45:886-95.

4. Fedewa AL, Ahn S. The effects of physical activity and physical fitness on children's achievement and cognitive outcomes: a metaanalysis. Res Q Exerc Sport 2011;82:521-35.

5. Ommundsen $\mathrm{Y}$, Løndal K, Loland S, . et alSport, children and wellbeing. In: Ben-Arieh A, Casas F, Frønes I, Korbin JE, . eds. Handbook of child well-being. Dordrecht: Springer Reference, 2014.

6. Waters E, de Silva-Sanigorski A, Hall BJ, et al. Interventions for preventing obesity in children. Cochrane database of systematic reviews 2011;12:CD001871.

7. Gadamer H. Truth and method. London: Sheed and Ward, 1989.

8. Pellegrini AD, Smith PK. Physical activity play: the nature and function of a neglected aspect of playing. Child Dev 1998;69:577-98

9. Gallahue DL, Ozmun JC. Understanding motor development: infants, children, adolescents, adults. . 6th ed. Boston: London: McGraw Hill, 2006:xix.

10. Caspersen CJ, Powell KE, Christenson GM. Physical activity, exercise, and physical fitness: definitions and distinctions for healthrelated research. Public Health Rep 1985;100:126-31.

11. Warren JM, Ekelund U, Besson $\mathrm{H}$, et al. Assessment of physical activity - a review of methodologies with reference to epidemiological research: a report of the exercise physiology section of the European Association of Cardiovascular Prevention and Rehabilitation. Eur J Cardiovasc Prev Rehabil 2010;17:127-39.

12. Dobbins M, Husson H, DeCorby K, et al. School-based physical activity programs for promoting physical activity and fitness in children and adolescents aged 6 to 18. Cochrane Database Syst Rev 2013;2:CD007651.

13. The Norwegian Directorate of Health. Anbefalinger om kosthold, ernæring og fysisk aktivitet (Norwegian guidelines on diet, nutrition and physical activity). Oslo: The Norwegian Directorate of Health, 2014.

14. Beets MW, Beighle A, Erwin HE, et al. After-school program impact on physical activity and fitness: a meta-analysis. Am J Prev Med 2009;36:527-37.

15. Beets MW, Weaver RG, Turner-McGrievy G, et al. Making policy practice in afterschool programs: a randomized controlled trial on physical activity changes. Am J Prev Med 2015;48:694-706.

16. Cradock AL, Barrett JL, Giles CM, et al. Promoting physical activity with the out of School Nutrition and physical activity (OSNAP) Initiative: a Cluster-Randomized Controlled Trial. JAMA Pediatr 2016;170:155-62.

17. Gortmaker SL, Lee RM, Mozaffarian RS, et al. Effect of an afterschool intervention on increases in children's physical activity. Med Sci Sports Exerc 2012;44:450-7.

18. Hughey SM, Weaver RG, Saunders R, et al. Process evaluation of an intervention to increase child activity levels in afterschool programs. Eval Program Plann 2014;45:164-70.

19. Green K. Understanding physical education. . London: Sage, 2008:280.

20. Løndal K. Revelations in bodily play. A study among children in an after-school programme. Oslo, 2010. Phd Dissertation.

21. Løndal K, Bergsjø C. Fysisk aktivitet i skolefritidsordningen: En undersøkelse $i$ fire skolefritidsordninger i Oslo. (Physical activity in after-school programmes. A study of four after-school programmes in Os/o). Oslo: Oslo Univeristy College, 2005.

22. Løndal K. Children's lived experience and their sense of coherence: Bodily play in a Norwegian after-school programme. Child Care in Practice 2010;16:391-407.

23. Løndal K, Lund S, Bergsjø CH. Tilrettelegging for fysisk aktivitet i skolefritidsordningen i Oslo. (Facilitating physical activity in after-school programmes in Oslo). Tidsskriftet FoU i praksis 2016;10:43-61.

24. Dowda M, Pate RR, Trost SG, et al. Influences of preschool policies and practices on children's physical activity. $J$ Community Health 2004;29:183-96.

25. Constantinides P, Montalvo R, Silverman S. Teaching processes in elementary physical education classes taught by specialists and nonspecialists. Teach Teach Educ 2013;36:68-76.

26. Løndal K, Greve A. Didactic approaches to Child-Managed Play: analyses of Teacher?s interaction styles in kindergartens and After-School Programmes in Norway. International Journal of Early Childhood 2015;47:461-79.

27. Cheon SH, Reeve J, Yu TH, et al. The teacher benefits from giving autonomy support during physical education instruction. J Sport Exerc Psychol 2014;36:331-46.

28. The Norwegian Directorate of Health. . Utviklingsstrategi for helsestasjons- og skolehelsetjenesten. (Strategy for the public health center- and the school health service). Rapport IS-1798. Os/o: The Norwegian Directorate of Health 2010.

29. Craig P, Dieppe P, Macintyre S, et al. Developing and evaluating complex interventions: the new Medical Research Council guidance. BMJ 2008;337:a1655.

30. Øygardslia H. Tiltak for økt fysisk aktivitetsnivå i skolefritidsordninger: En forskningsoppsummering. (Interventions to increase physical activity in after-school programmes; a systematic review). Master thesis. ODA Open Digital Archive: Oslo and Akershus University College of Applied Sciences, 2016.

31. Huberty JL, Dinkel DM, Beets MW. Evaluation of GoGirlGo!; A practitioner based program to improve physical activity. BMC Public Health 2014;14:118.

32. Iversen CS, Nigg C, Titchenal CA. The impact of an elementary after-school nutrition and physical activity program on children's fruit and vegetable intake, physical activity, and body mass index: Fun 5. Hawaii Med J 2011;70(7 Suppl 1):37-41.

33. Thaw JM, Villa M, Reitman D, et al. Evidence-based fitness promotion in an afterschool setting: implementation fidelity and its policy implications. New Dir Youth Dev 2014;2014:103-31.

34. Ryan RM, Deci EL. Active human nature. In: Hagger MS, Chatzisarantis NL, eds. Intrinsic motivation and self-determination in exercise and sport. Champaign: Human Kinetics, 2007:1-21.

35. Standage M, Gillison F, Treasure D. Self-determination and motivation in physical education. In: Hagger MS, Chatzisarantis NLD, eds. Intrinsic motivation and self-determination in exercise and sports. Champaign; IL: Human Kinetics, 2007:71-87.

36. Ryan RM, Deci EL. Self-determination theory and the facilitation of intrinsic motivation, social development, and well-being. Am Psychol 2000;55:68-78.

37. Ryan RM, Patrick H, Deci EL, et al. Facilitating health behaviour change and its maintenance: interventions based on SelfDetermination Theory. The European Helath Psychologist 2008;10:2-5. 
38. Edmunds J, Ntoumanis N, Duda JL. Testing a self-determination theory-based teaching style intervention in the exercise domain. Eur $J$ Soc Psychol 2008;38:375-88.

39. Broström S. SFO som arena for læring. In. Ankerstjerne IT, . SOog fritidspedagogik -før, nu og i fremtiden. Fredrikshavn: Dafolo, 2010.

40. Fortier MS, Duda JL, Guerin E, et al. Promoting physical activity: development and testing of self-determination theory-based interventions. Int J Behav Nutr Phys Act 2012;9:20.

41. Edmunds JK, Ntoumanis N. Perceived autonomy support and psychological need satisfaction in exercise. In: Hagger MS Chatzisarantis NLD, eds. Intrisic motivation and self-determination in exercise and sport. Champaign, IL: Human Kinetics, 2007.

42. Reeve J. Self-determination theory applied to educational settings. In: Deci EL, Ryan RM, eds. Handbook of self-determination research. Rochester, NY: University of Rochester Press, 2002.

43. The Norwegian Directorate of Health, , , . Fysisk aktivitet blant 6-, 9og 15-åringer i Norge. Resultater fra en kartlegging i 2011. (Physical activity among 6-,9- and 15 year olds in Norway 2011). IS-2002 Os/o; Helsedirektoratet, 2012.

44. Samdal O, Leversen I, Torsheim T, et al. Trender $i$ helse og livsstil bant barn og unge 1985-2005. (Trends in health and lifestyle among children and adolescents 1985-2005. Norwegian results from the study "Health behavior among school children. A WHO study in several countries"). Bergen: HEMIL-senteret, University of Bergen, 2009.

45. The KIDSCREEN Group Europe. The KIDSCREEN questionnaires. Quality of life questionnaires for children and adolescents Lengerich. Germany: Pabst Science Publishers, 2006.
46. Cole TJ, Bellizzi MC, Flegal KM, et al. Establishing a standard definition for child overweight and obesity worldwide: international survey. BMJ 2000;320:1240-3.

47. Broeck $A$, Vansteenkiste $\mathrm{M}$, Witte $\mathrm{H}$, et al. Capturing autonomy, competence, and relatedness at work: construction and initial validation of the Work-related basic need satisfaction scale. J Occup Organ Psychol 2010;83:981-1002.

48. Gagné M, Forest J, Vansteenkiste $\mathrm{M}$, et al. The multidimensional work motivation Scale: validation evidence in seven languages and nine countries. European Journal of Work and Organizational Psychology 2015;24:178-96 .

49. Andersen IA, Andersen JR. Validering av eit måleinstrument for jobbtilfredsheit. (Validation of an instrument to measure job satisfaction). Sykepleien Forskning 2012;7:334-40.

50. Pavot W, Diener E. Review of the satisfaction with Life Scale. Psychol Assess 1993;5:164-72.

51. Atkins S, Odendaal W, Leon N, et al. Qualitative process evaluation for complex interventions. Hallberg IR DAR, ed. Complex interventions in health; An overview of resarch methods. New York: Routhledge, 2015.

52. Kvale S, Rygge J, Brinkmann S, et al. Det kvalitative forskningsintervju. 2. utg. ed. Oslo: Gyldendal akademisk, 2009

53. Malterud K. Systematic text condensation: a strategy for qualitative analysis. Scand J Public Health 2012;40:795-805.

54. Schreier M. Qualitative Content analysis in practice. Thousand Oaks, CA: Sage Publications LTD, 2012.

55. Hallberg IR. Knowledge for health care practice. In: Richards DA, Hallberg IR, eds. Complex interventions in health: an overview of research methods. New York: Routhledge, 2015. 